\title{
UM ESTUDO PARA COMPREENDER A PREFERÊNCIA E ACEITAÇÃO DE CONSUMIDORES DE PURÊS DE PÊSSEGOS BRASILEIROS ${ }^{1}$
}

\author{
RICARDO PERAÇA TORALLES ${ }^{2}$, MARCELO BARBOSA MALGARIM $^{3}$, JOÃO LUIZ VENDRUSCOLO ${ }^{4}$, \\ RUFINO FERNANDO FLORES CANTILLANO ${ }^{5}$, ROSA DE OLIVEIRA TREPTOW ${ }^{6}$
}

\begin{abstract}
RESUMO - Testes de preferência e de aceitação foram realizados para determinar a relação entre parâmetros e atributos de qualidade de purês de pêssegos elaborados a partir das cvs. Granada, Jade, Esmeralda, Maciel, Eldorado, Jubileu, Br-6 e Magno. Pêssegos da safra 2003-2004 foram colhidos em pomar comercial na região de Pelotas - RS. As propriedades físicas e químicas foram determinadas com quatro repetições de vinte pêssegos de cada cultivar. O purê de pêssego foi elaborado por branqueamento contínuo. Os testes de preferência e aceitação foram aplicados com 50 consumidores, e as respostas dos consumidores foram registradas, respectivamente, em uma escala auto-ajustável e uma hedônica de nove pontos. Também, utilizou-se um teste descritivo com nove julgadores treinados. Analisaram-se os resultados por ANOVA, e as diferenças significativas, pelo teste de Tukey. As oito cultivares selecionadas variaram amplamente em suas características físicas e químicas. De modo geral, o teor de fenóis incrementou com o ciclo de colheita, e as cultivares Eldorado, BR-6, Jubileu e Magno foram superiores para relação SS/AT (e" 15,1), que é considerado um bom indicador para fruta de alta qualidade. Os purês elaborados com as cultivares Jubileu e Eldorado foram os preferidos pelos consumidores. O principal motivo dessa escolha foi o equilíbrio existente entre ácido e doce, que foi significativamente relacionado com SS/AT. As cultivares com baixo conteúdo de fenóis totais, quando manufaturadas, resultaram em purês de pêssegos de coloração amarela, que é uma característica desejável.
\end{abstract}

Termos de Indexação: Parâmetros e atributos de qualidade, preferência de consumidor e cor característica de purês de pêssegos.

\section{A STUDY TO UNDERSTAND THE CONSUMERS PREFERENCE AND ACCEPTANCE OF BRAZILIAN PEACHE PURÉES}

\begin{abstract}
Preference and acceptance tests were performed to determine the relationship between quality parameters and attributes of peach purées from cultivars Granada, Jade, Emeralda, Maciel, Eldorado, Jubileu, Br-6 and Magno. Peaches of the crop 2003/2004 were picked from a commercial orchard in Pelotas/RS. The physical and chemical properties were determined with four replications of twenty fruit each. The peach purées were manufactured by continuous blanching. Preference and acceptance tests were performed in peach purées with 50 consumers and the consumer's response were recorded using self-adjusting and nine-point hedonic scales respectively. In addition, the descriptive test was with nine trained judges. The results were analyzed by ANOVA, and significant differences were determined using Tukey's test. The physical and chemical characteristics of the eight cultivars of peach varied widely between themselves. In general, the total phenols increased with the crop cycle. The cvs. Eldorado, BR-6, Jubileu and Magno were superiors to SS/TA ratio (e" 15.1) that is a good indicator of high-quality fruit. Peach purées of Jubileu and Eldorado were the preferred of consumers. The main reason of this choice was the balance between sweet and acid that was significantly related to SS/TA. The peach cultivars with low total phenols contents, when the fruits were manufactured, had as results a yellow coloration desirable for peach purée.
\end{abstract}

Index Terms: Quality parameters and attributes, consumer preference, and characteristic color of peach pureé.

\section{INTRODUÇÃO}

A produção de pêssego, na Região Sul - RS, da safra (2003 2004) foi estimada em 50 mil toneladas, sendo essa região responsável por $50 \%$ da produção nacional. Entre as cultivares mais plantadas na região destacam-se a Granada, Jade, Esmeralda, Maciel, Eldorado, Jubileu, Br-6 e Magno, todas de caroço aderido (Medeiros \& Raseira, 1998).

Os estudos que determinam a qualidade em pêssegos in natura, citam aparência da cor da epiderme, flavor, voláteis, textura, sólidos solúveis (SS) e acidez total (AT) como os fatores mais importantes (Sistrunk, 1985; Meredith et al., 1989; Robertson et al., 1990; Cheng \& Crisoto, 1995; Crisoto \& Watkins, 1998). Recentemente, um programa californiano, preocupado em aumentar o estagnado consumo de pêssegos no EUA, $2 \mathrm{~kg}$ per capita-ano (Crisoto, 2002), desenvolveu, preliminarmente, um índice de qualidade mínimo para pêssegos, apontando significativa relação entre testes de aceitação realizados com consumidores e os parâmetros SS e AT (Crisoto \& Crisoto, 2003).

Para pêssegos destinados à elaboração de polpas, néctares e sucos são necessários parâmetros adicionais, capazes de predizer o seu comportamento durante e após o processamento, que possam estar relacionados com alteração da cor, sabor, aroma e textura (Ashurst, 1995; Garza et al., 2000; Toralles et al., 2004a; Versari et al., 2002). Também é necessário conhecer indicadores que revelem a preferência do consumidor (Sistrunk, 1985; Toralles et al., 2004b). O objetivo deste trabalho foi aplicar testes de preferência e de aceitação para determinar a relação entre parâmetros e atributos de qualidade de purês de pêssegos elaborados a partir das cultivares Granada, Jade, Esmeralda, Maciel, Eldorado, Jubileu, Br-6 e Magno.

\section{MATERIAL E MÉTODOS}

\section{Frutas}

Pêssegos da safra 2003 - 2004 de oito diferentes cultivares: Granada, Jade, Esmeralda, Maciel, Eldorado, Jubileu, Br-6 e Magno, foram colhidos em pomar comercial da região de Pelotas - RS. Selecionaram-se frutos sem defeitos e de aspecto maduro, os quais foram posteriormente armazenados por sete dias em câmara fria com temperatura entre $3-5^{\circ} \mathrm{C}$ e UR de 90 a $95 \%$.

\section{Análises físicas e químicas}

As propriedades físicas e químicas foram determinadas com quatro repetições de 20 pêssegos de cada cultivar. O caroço e a

\footnotetext{
1 (Trabalho 180-2005). Recebido: 01-11-2005. Aceito para publicação: 06-09-2006.

${ }^{2}$ Departamento de Ciência e Tecnologia de Alimentos, Universidade Federal de Pelotas, Pelotas-RS; e-mail: torashow@ cefetrs.tche.br;CP 354, CEP 96010900, Pelotas-RS, Brasil.

${ }^{3}$ Departamento de Fitotecnia/FAEM/UFPEL; e-mail:malgarim@ufpel.tche.br

${ }^{4},{ }^{5} \mathrm{e}^{6}$ EMBRAPA Clima Temperado, KM 78, BR 392, CP 403; 960001-970, Pelotas-RS, Brasil; vendrusc@cpact.embrapa.br; fcantill@cpact.embrapa.br; rosatreptow@hotmail.com
} 
casca foram removidos de cada pêssego, e um suco foi obtido da polpa moída em triturador centrífugo (Wallita). As análises dos sucos foram feitas em duplicata. O peso foi determinado através de uma balança Mettler com uma sensibilidade de 0,01g. A acidez titulável (AT), ácido ascórbico, sólidos solúveis (SS), açúcares redutores e totais foram determinados segundo metodologia da AOAC (2000). Os fenóis totais foram analisados segundo o método de Singleton \& Rossi (1965). A coloração dos frutos foi determinada através de colorímetro Minolta CR-300 calibrado com prato $(\mathrm{L}=96,98$, a $=+0,26$, $\mathrm{b}=+1,78)$. Os dados de cor foram convertidos em ângulo de cor, $\mathrm{q}=\tan$ 'b/a (Meredith et al., 1989). A firmeza, expressa em Newton (N), foi medida na parede do fruto, utilizando penetrômetro McCormick com ponta de $8 \mathrm{~mm}$. Cada medida resultou da média de duas leituras em lados opostos da secção equatorial das frutas. A coloração dos purês foi determinada através de colorímetro Hunter, Digimeter D25-2, calibrado com prato $(\mathrm{L}=92,4, \mathrm{a}=-1,0, \mathrm{~b}=+1,0)$.

\section{Preparo dos purês}

O processamento foi conduzido na planta-piloto da Universidade Federal de Pelotas-CAVG. As frutas foram selecionadas, lavadas, descaroçadas e descascadas utilizando o mesmo procedimento para o processamento de pêssegos em calda. Posteriormente, branquearam-se as frutas em um termoinativador enzimático tubular contínuo com controle da temperatura da jaqueta $(\mathrm{Tj})$, da temperatura de referência da polpa (Tp) e do tempo de branqueamento $(\mathrm{t})$. As condições de operação foram: $\mathrm{Tj}=95^{\circ} \mathrm{C} ; \mathrm{Tp}=$ $75^{\circ} \mathrm{C}$ e $80^{\circ} \mathrm{C} ; \mathrm{t}=4$ minutos. Após o branqueamento, nenhuma atividade residual foi detectada. Em seguida, as frutas passaram por despolpadeira com tela inox de 1,0 $\mathrm{mm}$ de abertura, seguido de uma segunda com abertura de $0,5 \mathrm{~mm}$. As amostras de purês foram fechadas assepticamente em latas e congeladas $-20^{\circ} \mathrm{C}$ para posterior avaliação sensorial. As amostras foram previamente descongeladas para aplicação do teste sensorial. Para tal finalidade, utilizou-se um banho termostatizado com controle de temperatura a $25^{\circ} \mathrm{C}$.

\section{Análise sensorial} distintas:

A análise sensorial dos purês foi realizada em duas etapas

Primeiramente, aplicou-se um teste de preferência com uma equipe de 50 consumidores. Utilizou-se, segundo Gay \& Mead (1992), uma escala auto-ajustável. Também, solicitou-se que descrevessem o motivo da sua preferência ou não pelo purê em avaliação. Através de uma escala hedônica de nove pontos, obteve-se o grau de aceitação dos purês em estudo (Meilgaard et al., 1999).

Posteriormente, realizou-se um teste descritivo para avaliação de atributos segundo ABNT (1993), com uma equipe constituída de nove julgadores treinados, que avaliaram as características de acidez, doçura, adstringência e sabor, fazendo uso de escalas nãoestruturadas de $9 \mathrm{~cm}$, cujo extremo esquerdo corresponde a menor intensidade (não perceptível) e o extremo direito, a maior intensidade (forte).

\section{Análise estatística}

Os resultados foram analisados por análise de variância (ANOVA), e as diferenças significativas entre as médias foi feita através do teste de Tukey $(\mathrm{p}<0,05)$. Quando aplicável, os resultados foram correlacionados $(\mathrm{p}<0,05)$. O programa estatístico utilizado foi o 'STATISTICA software' (Statsoft, 1996).

\section{RESULTADOS E DISCUSSÃO}

\section{Características físicas e químicas}

As oito cultivares selecionadas para o estudo variaram amplamente em suas características físicas e químicas (Tabelas $1 \mathrm{e}$ 2). Quanto à cor da epiderme, as características foram entre meiomaduro e maduro, alto valor de 'a' em relação a 'b', indicando mais amarelo do que verde, ou seja, baixo ângulo Hue, com exceção da cultivar Maciel. Quanto ao peso, a cultivar jubileu foi significativamente inferior às demais $(\mathrm{p}<0,05)$, e esta característica tem implicações na produtividade e no custo de processamento. As cultivares Esmeralda, Jade e Eldorado tiveram uma firmeza maior do que as demais, e esta é uma importante característica física que pode estar relacionada com a textura de purês.

A cultivar Eldorado teve maior índice SS/AT $(22,26)$, seguido pela BR-6 $(18,30)$, Jubileu $(17,69)$ e Magno $(15,71)$. Essa relação é considerada um bom indicador para fruta de alta qualidade, quando SS/AT ${ }^{3}$ 15,1 (Deshpande \& Salunkhe, 1964; Meredith et al., 1989). Por outro lado, a relação SS/AT, para cultivares precoces, foi significativamente inferior $(\mathrm{p}<0,05)$. Este comportamento também foi observado em cultivares precoces do Arkansas, EUA (Sistrunk \&

TABELA 1 - Características físicas em oito cultivares de pêssegos in natura. Pelotas-RS, safra 2003-2004.*

\begin{tabular}{|c|c|c|c|c|c|}
\hline Cultivares & $\begin{array}{l}\text { Período de } \\
\text { maturação }\end{array}$ & Peso (g) & $\mathrm{L}$ & Ângulo Hue & $\begin{array}{c}\text { Firmeza de } \\
\text { Polpa }(\mathrm{N})\end{array}$ \\
\hline Granada & $25 / 11-06 / 12$ & $137,97 \mathrm{bc}$ & $63,91 \mathrm{~cd}$ & $81,53 \mathrm{c}$ & $35,16 \mathrm{~cd}$ \\
\hline Esmeralda & $01 / 12-15 / 12$ & $128,58 \mathrm{~cd}$ & $62,66 \mathrm{~d}$ & $81,37 \mathrm{c}$ & $55,52 \mathrm{a}$ \\
\hline Jade & $07 / 12-20 / 12$ & $126,50 \mathrm{de}$ & $61,03 \mathrm{e}$ & $77,38 \mathrm{~d}$ & $50,62 \mathrm{ab}$ \\
\hline Maciel & $14 / 12-29 / 12$ & $154,08 \mathrm{a}$ & $67,14 \mathrm{~b}$ & 90,72 a & $46,23 \mathrm{~b}$ \\
\hline Eldorado & $27 / 12-06 / 01$ & 119,14 de & $64,91 \mathrm{c}$ & $75,31 \mathrm{~d}$ & $52,11 \mathrm{ab}$ \\
\hline Jubileu & $22 / 12-08 / 01$ & $84,42 \mathrm{f}$ & $68,68 \mathrm{a}$ & $83,44 \mathrm{bc}$ & $38,96 \mathrm{c}$ \\
\hline BR-6 & $21 / 01-28 / 01$ & $145,46 \mathrm{ab}$ & $69,92 \mathrm{a}$ & $83,80 \mathrm{bc}$ & $29,40 \mathrm{~d}$ \\
\hline Magno & $17 / 01-28-01$ & $118,41 \mathrm{e}$ & $65,25 \mathrm{c}$ & $86,68 \mathrm{~b}$ & $34,33 \mathrm{~cd}$ \\
\hline
\end{tabular}

*Valores médios com diferentes letras são significativos a $\mathrm{p}<0,05$ na mesma coluna.

TABELA 2 - Características químicas em oito cultivares de pêssegos in natura. Pelotas-RS, safra 2003-2004*.

\begin{tabular}{|c|c|c|c|c|c|c|}
\hline Cultivares & SS $\left({ }^{\circ}\right.$ Brix $)$ & $\begin{array}{l}\text { AT (\% ác. } \\
\text { cítrico) }\end{array}$ & SS/AT & $\begin{array}{c}\text { Vit. C } \\
\mathrm{mg} / 100 \mathrm{~mL}\end{array}$ & $\begin{array}{c}\text { Sacarose } \\
(\%)\end{array}$ & $\begin{array}{l}\text { Fenóis } \\
(\mu \mathrm{g} / \mathrm{g})\end{array}$ \\
\hline Granada & $9,05 \mathrm{f}$ & $0,86 \mathrm{~b}$ & $10,49 \mathrm{e}$ & $4,81 \mathrm{~d}$ & $5,75 \mathrm{c}$ & $404,57 \mathrm{e}$ \\
\hline Esmeralda & $12,57 \mathrm{~cd}$ & $1,02 \mathrm{a}$ & $12,34 \mathrm{~d}$ & $8,60 \mathrm{~b}$ & $8,42 \mathrm{~b}$ & $501,25 \mathrm{~d}$ \\
\hline Maciel & $12,10 \mathrm{de}$ & $0,87 \mathrm{~b}$ & $13,91 \mathrm{~d}$ & $6,48 \mathrm{c}$ & $8,22 \mathrm{~b}$ & $812,73 \mathrm{c}$ \\
\hline Eldorado & $16,73 \mathrm{a}$ & $0,75 \mathrm{c}$ & $22,27 \mathrm{a}$ & $11,80 \mathrm{a}$ & $9,58 \mathrm{a}$ & $923,71 \mathrm{~b}$ \\
\hline Jubileu & $14,25 \mathrm{~b}$ & $0,80 \mathrm{bc}$ & $17,70 \mathrm{~b}$ & $5,49 \mathrm{~cd}$ & $8,95 \mathrm{ab}$ & $405,02 \mathrm{e}$ \\
\hline BR-6 & $13,67 \mathrm{bc}$ & $0,75 \mathrm{c}$ & $18,30 \mathrm{~b}$ & $9,82 \mathrm{~b}$ & $7,91 \mathrm{bc}$ & $1169,10 \mathrm{a}$ \\
\hline Magno & $12,80 \mathrm{~cd}$ & $0,81 \mathrm{bc}$ & $15,71 \mathrm{c}$ & $12,04 \mathrm{a}$ & $7,91 \mathrm{bc}$ & $1180,20 \mathrm{a}$ \\
\hline
\end{tabular}

*Valores médios com diferentes letras são significativos a $\mathrm{p}<0,05$ na mesma coluna. 
Rom, 1976).

As cultivares Magno (12,04 mg.mL $\left.{ }^{-1}\right)$ e Eldorado (11,80 mg.mL 1) foram significativamente superiores às demais para vitamina $C$, que é cerca de cinco vezes menor que a quantidade encontrada na laranja (Mahan, Escott-Stump, 1999). Mesmo assim, a vitamina C é considerada um importante parâmetro de qualidade, não só devido ao seu valor nutricional, mas também devido as suas contribuições funcionais na qualidade do produto e que podem estar relacionado com algum atributo sensorial (Gil et al., 2002; Toralles et al., 2004b).

O incremento do teor de fenóis, de modo geral, acompanhou o ciclo de colheita, sendo a cultivar Jubileu uma exceção. A concentração de fenóis totais está relacionada com escurecimento enzimático e, recentemente, foi demonstrada através de estudo comparativo entre coeficiente de especificidade $\left(\mathrm{V}_{\max } / \mathrm{K}_{\mathrm{m}}\right)$ e fenóis totais (Toralles et al., 2004a).

\section{Coloração dos purês}

Os maiores valores de 'L', 'a', 'b' foram observados na cultivar Jade, de coloração alaranjada (Figura 1, Tabela 3). Os purês elaborados com as cultivares Granada, Esmeralda e Jubileu tiveram uma coloração amarela característica, como indicado pelo alto valor HUNTER para 'b' e 'L'. Já, os purês elaborados com as cultivares BR-6, Eldorado, Magno e Maciel tiveram uma coloração amarelo-escura, que é uma característica não-desejável em purês.

TABELA 3 - Coloração Hunter 'L', 'a' e 'b' dos oito purês*.

\begin{tabular}{lccc}
\hline & $\mathrm{L}$ & $\mathrm{a}$ & $\mathrm{b}$ \\
\hline Granada & $58,20 \mathrm{a}$ & $13,35 \mathrm{bc}$ & $32,00 \mathrm{bc}$ \\
Esmeralda & $57,35 \mathrm{ab}$ & $14,65 \mathrm{bc}$ & $32,25 \mathrm{ab}$ \\
Jade & $57,95 \mathrm{a}$ & $16,75 \mathrm{a}$ & $34,00 \mathrm{a}$ \\
Maciel & $52,50 \mathrm{c}$ & $12,75 \mathrm{c}$ & $27,25 \mathrm{f}$ \\
Eldorado & $54,95 \mathrm{bc}$ & $13,20 \mathrm{bc}$ & $29,55 \mathrm{de}$ \\
Jubileu & $56,85 \mathrm{ab}$ & $14,75 \mathrm{c}$ & $31,85 \mathrm{bc}$ \\
BR-6 & $54,70 \mathrm{bc}$ & $13,45 \mathrm{bc}$ & $30,25 \mathrm{~cd}$ \\
Magno & $52,90 \mathrm{c}$ & $13,40 \mathrm{bc}$ & $28,20 \mathrm{ef}$ \\
\hline *alores médios com diferentes letras são significativos a $\mathrm{p}<0,05$ na mesma
\end{tabular}
coluna

TABELA 4 - Coeficientes de correlação entre fenóis totais e coloração Hunter 'L', 'a' e 'b'.

\begin{tabular}{cccc}
\hline & $\mathrm{L}$ & $\mathrm{a}$ & $\mathrm{b}$ \\
Fenóis totais & $-0,84^{*}$ & $-0,53$ & $-0,72^{*}$ \\
\hline
\end{tabular}

$* \mathrm{p}<0,05$ e $\mathrm{n}=8$

TABELA 5 - Teste de preferência para consumidores de purê de pêssego.

\begin{tabular}{lc}
\hline Purês & Preferêcia $^{1,2}$ \\
\hline Granada & $191,0 \pm 1,7 \mathrm{bc}$ \\
Esmeralda & $212,0 \pm 1,9 \mathrm{bc}$ \\
Jade & $221,0 \pm 1,8 \mathrm{~b}$ \\
Maciel & $72,0 \pm 1,9 \mathrm{bc}$ \\
Eldorado & $319,0 \pm 2,0 \mathrm{a}$ \\
Jubileu & $352,0 \pm 1,3 \mathrm{a}$ \\
BR-6 & $191,0 \pm 2,1 \mathrm{bc}$ \\
Magno & $145,0 \pm 2,1 \mathrm{c}$ \\
\hline
\end{tabular}

${ }^{1}$ média \pm desvio.

${ }^{2}$ Avaliação sensorial de preferência através de um painel de 50 consumidores Utlizou-se uma escala auto ajustável. A magnitude dos valores é resultado da soma de ordens obtidos da escala. As diferenças observadas foram significativas $(\mathrm{p}<0,05)$ usando tabela de Newel e Mac Farlane (ABNT-NBR 13170, 1994).
Os coeficientes de correlações calculados para relação entre as médias dos valores Hunter de 'L', 'a', 'b' e fenóis totais estão na Tabela 4. Fenóis totais foram significativamente correlacionados $(p<0,05)$ com 'L' e 'b', mas não com 'a'. O valor negativo observado para os coeficientes de correlação indica que a correlação foi inversa, ou seja, o incremento do valor dos fenóis totais é acompanhado do decréscimo de 'L'e 'b'.

\section{Preferência e aceitação de consumidores}

Os purês elaborados com as cultivares Eldorado e Jubileu tiveram maior preferência dos consumidores e foram significativamente diferente dos demais a $\mathrm{p}<0,05$ (Tabela 5). Fazendo uso de uma escala hedônica de 9 pontos, os consumidores registraram o quanto gostaram ou desgostaram das amostras e praticamente se obteve a mesma ordenação (Figura 2). Anteriormente, fora observado, conforme Tabela 2, que Jubileu e Eldorado foram as cultivares com maiores valores para SS e SS/AT.

Os consumidores indicaram o equilíbrio entre doce e ácido como o motivo mais importante para a escolha do purê, seguido de sabor característico, doçura, textura e aroma (Figura 3).

O purê elaborado com a cultivar Magno foi indicado como o menos preferido, seguido do purê da cultivar Maciel, e a justificativa principal foi o residual amargo (Figura 4). Os maiores valores para fenóis totais foram observados nessas duas cultivares, e, provavelmente, o residual amargo pode estar relacionado com fenóis totais. Para 'L' e 'b' foi significativa a correlação, e o processamento térmico utilizado pode ter potencializado o residual amargo e a cor amarelo-escura observada nos purês elaborados com cultivares de maior teor de fenóis. Considerando que o sistema enzimático foi previamente inativado, pode-se dizer que o processamento térmico induziu alterações de origem 'não-enzimática', principalmente de compostos fenólicos. Recentes investigações com pêssegos têm sido feitas nessa direção (Tourjee et al., 1998; Asami et al., 2002; Hong et al., 2004).

\section{Intensidade dos atributos sensoriais (teste descritivo)}

$\mathrm{Na}$ Tabela 6, é feita uma análise comparativa da intensidade dos atributos doçura, acidez, sabor e adstringência para os purês das oito cultivares. As diferenças entre as médias das intensidades foram significativas pelo teste de Tukey $(\mathrm{p}<0,05)$. Para doçura, as maiores intensidades foram observadas em purês das cultivares Magno e Maciel, sendo classificadas como moderadamente doce; com doçura regular os purês de Eldorado, Br-6 e Jubileu e, com ligeira doçura, os purês de Jade, Esmeralda e Granada. A acidez teve um comportamento inverso, com os purês das cultivares Esmeralda, Granada e Jade classificados como muito ácidos e, com regular acidez, os purês de Jubileu, BR-6 e Magno. Com relação ao sabor característico, os purês de Jubileu e Eldorado foram classificados com sabor intenso; Esmeralda, BR-6 e Maciel com sabor moderado; Granada com sabor regular e os demais purês, Magno e Jade, com

TABELA 6 - Análise comparativa da intensidade dos atributos sensorias doçura, acidez, sabor característico e adstringência de purês de pêssegos*.

\begin{tabular}{lcccc}
\hline & Doçura & Acidez & $\begin{array}{c}\text { Sabor } \\
\text { característico }\end{array}$ & Adstringência \\
\hline Granada & $1,47 \mathrm{f}$ & $7,87 \mathrm{~b}$ & $4,67 \mathrm{~d}$ & $0,24 \mathrm{f}$ \\
Esmeralda & $2,25 \mathrm{e}$ & $8,54 \mathrm{a}$ & $6,38 \mathrm{c}$ & $1,35 \mathrm{~d}$ \\
Jade & $2,86 \mathrm{~d}$ & $7,11 \mathrm{c}$ & $2,13 \mathrm{e}$ & $0,13 \mathrm{f}$ \\
Maciel & $8,18 \mathrm{a}$ & $1,86 \mathrm{~g}$ & $5,85 \mathrm{c}$ & $1,57 \mathrm{~d}$ \\
Eldorado & $7,51 \mathrm{~b}$ & $3,07 \mathrm{f}$ & $7,13 \mathrm{~b}$ & $3,43 \mathrm{c}$ \\
Jubileu & $5,84 \mathrm{c}$ & $6,04 \mathrm{~d}$ & $8,24 \mathrm{a}$ & $0,83 \mathrm{e}$ \\
BR-6 & $6,32 \mathrm{c}$ & $5,06 \mathrm{e}$ & $6,25 \mathrm{c}$ & $5,20 \mathrm{~b}$ \\
Magno & $8,55 \mathrm{a}$ & $1,43 \mathrm{~g}$ & $1,52 \mathrm{e}$ & $6,11 \mathrm{a}$ \\
\hline *valores médios com diferentes letras são significativos a p<0,05 na mesma
\end{tabular}

coluna. 
TABELA 7 - Coeficientes de correlação entre atributos sensorias e SS, AT, SS/AT, sacarose, ácido ascórbico e fenóis totais.

\begin{tabular}{lcccc}
\hline & Doçura & Acidez & Sabor & Adstringência \\
\hline SS & $0,60^{*}$ & $-0,42^{*}$ & $0,48^{*}$ & - \\
AT & $-0,51^{*}$ & $0,46^{*}$ & $-0,05$ & - \\
SS/AT & $0,64^{*}$ & $-0,49^{*}$ & $0,41^{*}$ & - \\
Sacarose & $0,49^{*}$ & $-0,33$ & $0,38^{*}$ & - \\
Vitamina C & - & - & $-0,30$ & $0,72^{*}$ \\
Fenóis totais & - & - & $-0,11$ & $0,95^{*}$ \\
\hline
\end{tabular}

$* \mathrm{p}<0,05$ e $\mathrm{n}=32$

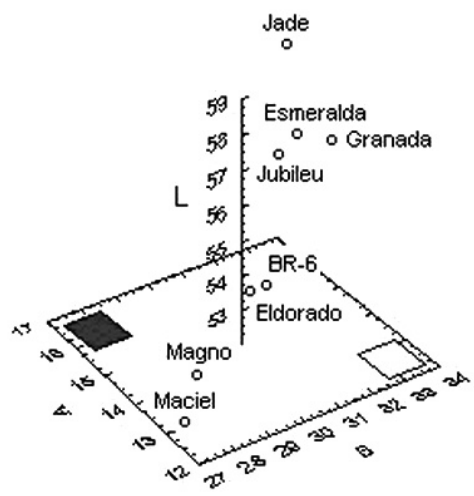

FIGURA 1 - Valores de L, 'a' e 'b' obtidos em colorímetro hunter para oito cultivares de pêssegos.

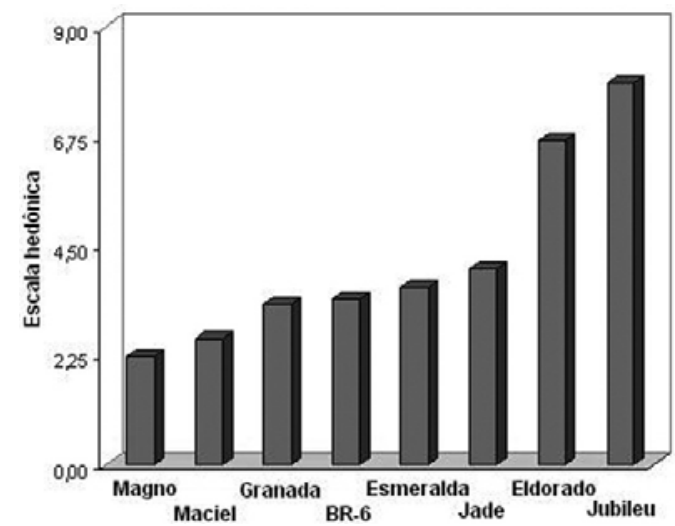

FIGURA 2 - Valores hêdonicos obtidos na avaliação de purês de pêssego.

ligeiro sabor característico. Quanto à adstringência, as maiores intensidades foram observadas nos purês das cultivares Magno e BR-6, com regular e moderada adstringência; Eldorado com ligeira adstringência, e, nas demais, os julgadores não perceberam.

Significativa correlação $(\mathrm{p}<0,05)$ foi observada entre as relações SS, AT e SS/AT com os atributos doçura e acidez, sendo a melhor correlação entre SS/AT e doçura (Tabela 7). Estas interações mostraram que o equilíbrio entre doce e ácido, principal motivo na escolha dos purês pelos consumidores, também está relacionado com SS/AT. O sabor, segundo em preferência pelos consumidores, manteve baixa correlação com SS e sacarose. Por outro lado, fenóis totais e ácido ascórbico foram significativamente correlacionados $(\mathrm{p}<0,05)$ com adstringência, mas não com sabor. A relação entre fenóis totais, adstringência do mesocarpo e flavor já foram citados por Craft (1961), Li et al.(1972), Senter et al. (1989) e Tomas-Barberan et al. (2001). A precisa relação dessas interações necessita de investigações adicionais, principalmente para desvendar os componentes do sabor característico.

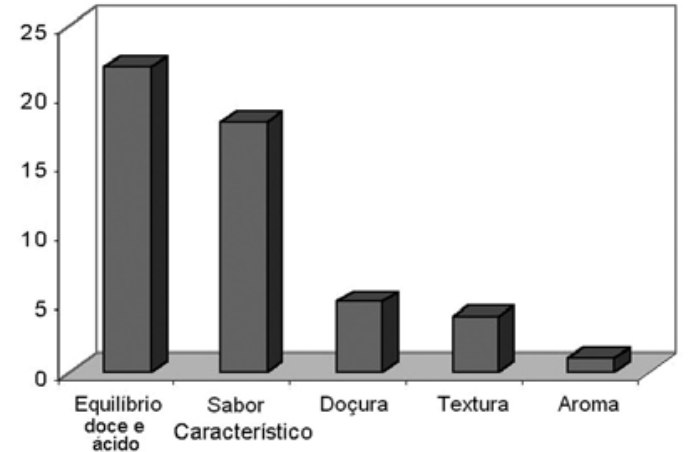

FIGURA 3 - Atributos de preferência para purês de pêssego.

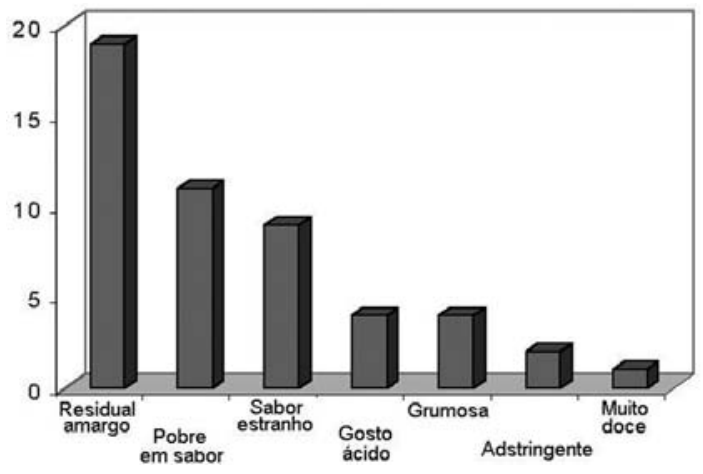

FIGURA 4 - Atributos de rejeição para purês de pêssego.

\section{CONCLUSÕES}

Os purês elaborados a partir das cultivares Jubileu e Eldorado foram os preferidos por consumidores, sendo os que apresentaram valores maiores para relação SS/AT. Os atributos sensoriais doçura e acidez estão relacionados com a relação SS/AT, enquanto fenóis totais e vitamina $\mathrm{C}$ com adstringência. A coloração amarela, característica e desejável, para purês de pêssegos, indicado por altos valores de Hunter para ' $L$ ' e 'b', foi alcançada quando os purês foram elaborados com as cultivares Jade, Esmeralda, Granada e Jubileu, todas com baixo conteúdo de fenóis totais.

\section{REFERÊNCIAS}

AOAC - Official Methods of Analysis of AOAC International/Dr Willian Horwitz (ed)-17. ed. Maryland: AOAC International, 2000. 818p.

ASSOCIAÇÃO BRASILEIRA DE NORMAS TÉCNICAS. Métodos de análise sensorial dos alimentos e bebidas. NBR 12994. Rio de Janeiro, 1993.

ASSOCIAÇÃO BRASILEIRA DE NORMAS TÉCNICAS. Teste de ordenação em análise sensorial. NBR 13170. Rio de Janeiro, 1994.

ASAMI, D.K.; HONG, Y.J.; BARRET, D.M.; MITCHELL, A.E. Processing-induced changes in total phenolics and procyanidins in clingstone peaches. Journal of the Science of Food and Agriculture. v. 83, p. 56-63, 2002.

ASHURST, P.R. Production and Packaging of Non-Carbonated Fruit Juices and Fruit Beverages. 2a. ed. London: Blackie Academic, 1995.429p.

CHENG, G.W.; CRISOSTO, C.H. Browning potential, phenolic composition, and polyphenoloxidase activity of buffer extracts of peach and nectarine skin tissue. Journal of the American Society of Horticultural Science, v. 120, n. 5, p. 835-838, 1995.

CRAFT, C.C. Polyphenolic compounds in Elberta Peaches during storage and ripening. American Society for Horticultural Science, v. 78, p. 119-131, 1961. 
CRISOTO, C.H. How do we increase peach consumption? Acta Horticulturae, v. 592, p. 601-605, 2002.

CRISOTO, G.M.; WATKINS, M. Chemical and organoleptic description of white flesh nectarines and peaches. Acta Horticulturae, v. 465, p. 497-505, 1998.

CRISOTO, G.M.; CRISOTO C.H. Understanding consumer acceptance of peach, nectarine, and plum cultivars. Acta Horticulturae, v. 604, p. 115-119, 2003.

DESHPANDE, P.B.; SALUNKHE, D.K. Effect of maturity and storage on certain biochemical changes in apricots and peaches. Food Technology, v. 18, n. 8, p. 85-88, 1964.

GARZA, S; IBARAZ, A.; PAGAN, J; GINER, J. Non-enzymatic in peach purée during heating. Food Research International, v.32, n. 5, p. 335-343. 2000.

GAY, C.; MEAD, R.A. A statistical appraisal of the problem as sensory measurement. Journal of Sensory Studies, v.7, p. 205-207, 1992.

GIL, M. I.; TOMAS-BARBERAN, F. A.; HESS-PIERCE, B.; KADER, A. Antioxidant capacities, phenolic compounds, carotenoids, and vitamin $\mathrm{C}$ contents of nectarine, peach, and plum cultivars from California. Journal of Agricultural and Food Chemistry , v.50, n. 17, p.4976-4982, 2002.

HONG, YJ.; BARRETT, D.M.; MITCHELL, A.E. Liquid chromatography/mass spectrometry investigation of the impact of thermal processing and storage on peach procyanidins. Journal Agricultural and Food Chemistry, v. 52, p. 2366-2371, 2004.

LI, K.C.; BOGGESS, T.S.; HEATON, B. Relationship of sensory ratings with tannin components of canned peaches. Journal of Food Science. v.37, p.177-178, 1972.

MAHAN, L. K.; ESCOTT-STUMP, S. Krause: alimentos, nutrição e dietoterapia. 9. ed. São Paulo: Roca, 1998. 1.179p.

MEDEIROS, C.A.; RASEIRA, M.C. A cultura do pessegueiro. Brasilia: Embrapa SPI, 1998.350p.

MEILGAARD, M.; CIVILLE, G.V.; CARR, B.T. Sensory evaluation tecniques. 2 ed Boca Raton: CRC Press, 1999, 281p.

MEREDITH, F.I.; ROBERTSON, J.A.; HOVART, R.J. Changes in physical and chemical parameters associated with quality and postharvest ripening of Harvester peaches. Journal Agricultural and Food Chemistry, v. 37, n. 5, p. 1.210-1.214, 1989.

ROBERTSON, J.A.; MEREDITH, F.I.; HOVART, R.J.; SENTER, S.D. Effect of cold storage and maturity on the physical and chemical characteristics and volatile constituents of peach $(\mathrm{Cv}$. Cresthaven) Journal Agricultural and Food Chemistry, v. 38, p. 620-624, 1990.
STAT SOFT, Inc. (1996). STATISTICA for Windows (Computer program manual). Tolsa, OK: StatSoft, Inc., 2300 East 14 th Street, Tulsa, OK 74104, phone: (918) 749-1119, fax: (918) 749-2219, email: info@statsoftinc.com, WEB: http://www.statsoftinc.com.

SENTER, S.D.; ROBERTSON, J.A.; MEREDITH, F.I. Phenolic compounds of the mesocarp of cresthaven peaches during storage and ripening. Journal of Food Science, v. 54, n. 5, p. 1.259-1.268, 1989.

SISTRUNK, W.; ROM, R.C. Quality attributes of peaches for processing. Arkansas Farm Research, v. 25, n. 3, p.1, 1976.

SISTRUNK, W.A. Peach quality assessment: Fresh and processed. In: Pattee, H.E. (ed.). Evaluation of Quality of Fruits and Vegetables. Westport, Connecticut, USA; AVI Publishing Co. p. $1-46,1985$.

SINGLETON, V.L.; ROSSI, J.A. Colorimetry of total phenolics with phosphomolybdic-phosphotungstic acid reagent. American Journal of Enology and Viticulture, v. 16, p. 144-158, 1965.

TOMAS-BARBERAN, F.A.; GIL, M.I.; CREMIN, P.; WATERHOUSE, A.L.; HESS-PIERCE, B.; KADER, A. HPLC-DAD-ESIMS analysis of phenolic compounds in nectarines, peaches, and plums. Journal of Agricultural and Food Chemistry, v. 49, n.10, p. 4.7484.760, 2001.

TORALLES, R.T.; VENDRUSCOLO, J.L.; HAAS, L.I.R.; FERRI, N.L.; DELPINO, F.A.V.; ANTUNES, P.L. Partial characterization of the enzymatic browning for polyphenoloxidase in peaches of the cv. Ganada, Jade, Esmeralda and Maciel. Revista Brasileira de Agrociência, v. 10, n. 2, p. 241-244, Jan-mar, 2004a.

TORALLES, R.P.; MALGARIM, M.B.; VENDRUSCULO, J.L.; CANTILHANO, F.R.F.; TREPTOW, R.O. Parâmetros e atributos de qualidade para o processamento de purês de pêssegos brasileiros. In: XIX CBCTA- CIÊNCIA E TECNOLOGIA DE ALIMENTOS: ESTRATÉGIAPARA O DESENVOLVIMENTO, 2004, Recife. Anais... Recife: CBCTA. 2004b.

TOURJEE, K.; BARRETT, D.M.; ROMERO, M.V.; GRADZIEL, T.M. Measuring flesh color variability among processing clingstone peach genotypes differing in carotenoid composition. Journal of the American Society of Horticultural Science, v. 123, n.3, p. 433-437, 1998.

VERSARI, A.; CASTELARI, M.; PARPINELLO, G.P.; RIPONI, C.; GALASSI, S. Characterisation of peach juices obtained from cultivars Redhaven, Suncrest and Maria Marta grown in Italy. Food Chemistry, v. 76, p. 181-185, 2002. 